\title{
Relación entre enfermedad periodontal, infección bacteriana ascendente y patología placentaria con parto prematuro
}

\author{
Alfredo 0 valle ${ }^{1}$, Jorge $G$ amonal ${ }^{2}$, M Angélica Martínez ${ }^{3}$, \\ Nora Silva ${ }^{4}$, Elena Kakarieka ${ }^{5}$, Ariel Fuentes ${ }^{1}$, \\ Alejandra Chaparro2 ${ }^{2}$ Marta G ajardo ${ }^{4}$, Rubén León ${ }^{6 a}$, \\ Alexis Ahumada ${ }^{2}$, Carlos Cisternas ${ }^{2}$. \\ Relationship between periodontal \\ diseases and ascending bacterial \\ infection with preterm delivery
}

Background: There is an association between periodontal diseases and preterm delivery. Aim: To assess the relationship between periodontal diseases, ascending bacterial infection and placental pathology with preterm delivery. Patients and methods: A periodontal examination and collection of amniotic fluid and subgingival plaque samples were performed in women with preterm labor with intact membranes, without an evident clinical cause or preterm premature rupture of membranes, without clinical chorioamnionitis or labor and a gestational age between 24 and 34 weeks. Microbial invasion of the amniotic cavity was defined as the presence of a positive amniotic fluid culture. Cervicovaginal infection was defined as a bacterial vaginosis or positive culture of cervix or vagina with a high neutrophil count. Ascending bacterial infection was diagnosed as the microbial invasion of the amniotic cavity by ascending bacteria or cervicovaginal infection. Corioamnionitis, funisitis or vellositis were diagnosed. Results: Fifty-nine women were included: fortytwo with preterm labor with intact membranes and seventeen with preterm premature rupture of membranes. The prevalence of periodontal diseases was 93.2\%. Microbial invasion of the amniotic fluid was detected in $27.1 \%$ of patients. Periodontal pathogenic bacteria were isolated in $18.6 \%$ of amniotic fluid samples and $71.2 \%$ of subgingival plaque samples. The prevalence of ascending bacterial infection was $83.1 \%$ and in $72.9 \%$ of women it was associated with periodontal disease. Preterm delivery ( $<37$ weeks) occurred in $64.4 \%$ of patients and was significantly associated with generalized periodontal disease and with the association of ascending bacterial infection and periodontal diseases. Patients with preterm delivery and generalized periodontal disease had a higher frequency of chorioamnionitis and funisitis. Conclusions: Generalized periodontal disease and its association with ascending bacterial infection are related to preterm delivery and placental markers of bacterial ascending infection (Rev Méd Chile 2009; 137: 504-14).

(Key words: Chorioamnionitis; Obstetric labor, premature; Periodontal diseases)

Recibido el 12 de mayo, 2008. Aceptado el 9 de enero, 2009.

Trabajo financiado por Proyectos de Investigación en Temas Nacionales DI 2003.

${ }^{1}$ Servicio y Departamento de Obstetricia, Ginecología y Neonatología, Hospital San Borja Arriarán, Facultad de Medicina, Universidad de Chile. ${ }^{2}$ Departamento de Odontología Conservadora, Facultad Odontología, Universidad de Chile. ${ }^{3}$ Programa de Microbiología, Instituto de Ciencias Biomédicas, Facultad de Medicina, Universidad de Chile. ${ }^{4}$ Departamento de Patología, Facultad de Odontología, Universidad de Chile. ${ }^{5}$ Servicio de Anatomía Patológica, Hospital San Borja Arriarán. ${ }^{6}$ Departamento de Bíoquímica, Facultad de Odontología, Universidad de Chile. Santiago de Chile.

aBioquímico

Correspondencia a: Alfredo Ovalle S. Servicio de Obstetricia, Ginecología y Neonatología, Hospital San Borja Amiarán. Santa Rosa 1234, Santiago, Chile. Fax: 562 2327565. E mail: aovalle@hotmail.com 
$\mathrm{E}$ parto prematuro complica actualmente en Estados Unidos de Norteamérica entre $12 \%$ y $13 \%$ y en Europa y países desarrollados entre $5 \%$ y $9 \%$ de las gestaciones ${ }^{1}$ y es la causa más importante de morbilidad y mortalidad perinatal en todo el mundo ${ }^{1,2}$. A pesar del conocimiento de los factores de riesgo y de los mecanismos relacionados con el parto prematuro y de la introducción de políticas de salud pública e intervenciones médicas, no se ha podido reducir este resultado adverso del embarazo ${ }^{1}$.

La enfermedad periodontal (gingivitis y periodontitis crónica) es uno de los factores de riesgo invocados últimamente para nacimientos prematuros. Se produce por un crecimiento anormal de patógenos periodontales en la placa subgingival, seguido de una respuesta inflamatoria del hospedero. Tanto la bacteria periodontal como la respuesta inmune desencadenada, pueden causar destrucción tisular y pérdida de piezas dentarias ${ }^{3}$.

La enfermedad periodontal compromete los sitios subgingivales y libera al torrente sanguíneo crónicamente bacterias generalmente anaerobias Gram negativas, productos bacterianos, endotoxinas, lipopolisacáridos y respuesta inflamatoria del hospedero, que diseminados por vía hematógena transplacentaria resultan en infección-inflamación intrauterina y parto prematuro ${ }^{4-6}$. La embarazada, por los cambios hormonales (estrógeno y progesterona) posee un mayor riesgo de desarrollar esta patología.

La severidad de esta enfermedad depende de la magnitud de la respuesta inflamatoria, principalmente factor de necrosis tumoral alfa, interleuquina 1 y 6, prostaglandina E2 y metaloproteinasas ${ }^{7,8}$. La mayor repuesta inflamatoria ocurre cuando la periodontitis clínicamente es severa o generalizada y en estos casos el riesgo de parto prematuro es mayor $6,9-11$.

Inicialmente se encontró que la relación con parto prematuro era independiente de las infecciones genitourinarias y de otros factores de riesgo ${ }^{6,12}$. Sin embargo, son escasas las publicaciones que se refieren a la asociación entre enfermedad periodontal e infección bacteriana ascendente (IBA) y su relación con parto prematuro.

Los hallazgos patológicos placentarios pueden ayudar a sugerir la participación de enfermedad periodontal o de IBA en el parto prematuro. La corioamnionitis histológica $(\mathrm{CH})$ y la funisitis son marcadores de IBA aguda y la vellositis, habitualmente lesión crónica, aparece de preferencia en las infecciones transplacentarias ${ }^{13-16}$.

El objetivo de este estudio fue determinar la relación entre enfermedad periodontal, IBA y patología placentaria, con parto prematuro.

\section{MATERIAL Y MÉTODOS}

Criterios de selección de pacientes. Embarazadas entre 24 y 34 semanas de gestación, ingresadas al Hospital Clínico San Borja Arriarán entre mayo de 2004 y diciembre de 2007, con el diagnóstico de trabajo de parto prematuro sin causa clínica evidente, con membranas intactas (MI) o con el diagnóstico de rotura prematura de membranas (RPM), fueron invitadas a participar en este estudio. El Comité de Ética aprobó la realización de este trabajo. Se obtuvo consentimiento informado de cada paciente. La Figura 1 describe el proceso de enrolamiento de pacientes.

La amniocentesis bajo guía ultrasonográfica para obtención de líquido amniótico (LA), la toma de muestras cervicovaginales y de la placa bacteriana subgingival, el diagnóstico clínico de enfermedad periodontal y el envío de placentas a estudio fueron requisitos indispensables de enrolamiento. Se incorporaron sólo pacientes admitidas durante la mañana de los días hábiles, porque los procedimientos descritos y el estudio microbiológico inmediato de LA, cervicovaginal y de la placa bacteriana subgingival realizados antes de administrar antibióticos a la madre, se pudieron hacer exclusivamente en estos horarios.

Se consideró como trabajo de parto prematuro sin causa clínica evidente la presencia de cuatro

\begin{tabular}{|lll|}
\hline Pacientes elegidas & $=$ & 595 \\
Pacientes no enroladas & $=$ & 532 \\
Pacientes enroladas & $=$ & 63 \\
Pacientes excluidas & $=$ & 4 \\
Cumplieron criterios de selección & $=$ & 59 \\
\hline
\end{tabular}

Figura 1. Diagrama de flujo de pacientes enroladas.

ARtíCULO DE 
contracciones o más cada 30 min objetivada con monitor por un período de $1 \mathrm{~h}$, además de dilatación cervical de 1 (permeable a 1 dedo) hasta $2 \mathrm{~cm}$ o cuello $25 \mathrm{~mm}$ o menos de longitud medido por ultrasonografía.

La rotura de membranas fue clínicamente documentada visualizando pérdida evidente de LA a través del orificio cervical con espéculo estéril o empleando la prueba de la nitrazina. La edad gestacional se determinó por historia menstrual confiable o por ultrasonografía realizada antes de las 24 semanas.

Criterios de exclusión. Uso de antibióticos 30 días previos, alergia a la clindamicina o gentamicina, hemorragia genital significativa, desprendimiento de placenta, placenta previa, malformación 0 muerte fetal, anomalías uterinas, cerclaje cervical, sufrimiento fetal, presencia de dispositivo intrauterino, gestación múltiple, infección ovular clínica (corioamnionitis clínica), síndrome hipertensivo durante el embarazo, colestasia intrahepática, diabetes pre y gestacional, alcoholismo y abuso de drogas, infección extrauterina, incluyendo pielonefritis aguda y restricción del crecimiento fetal intrauterino (< percentil 10 para la edad gestacional) ${ }^{17}$.

Estudio microbiológico ginecológico. Se obtuvieron muestras cervicales, vaginales y de LA para estudio microbiológico. La técnica en la toma de muestras y el diagnóstico de bacterias anaerobias facultativas y de anaerobias estrictas, así como el cultivo de micoplasmas urogenitales fueron realizados de acuerdo a métodos previamente descritos ${ }^{18-24}$ en el Laboratorio de Microbiología, I.C.B.M. Facultad de Medicina, Universidad de Chile. El diagnóstico de $\mathrm{C}$ trachomatis se hizo por amplificación de ADN (RPC). El diagnóstico microbiológico de vaginosis bacteriana (VB) se realizó evaluando la tinción de Gram del flujo vaginal según el método propuesto por Nugent y cols $^{25}$. Invasión microbiana de la cavidad amniótica (IMCA) fue definida por el cultivo positivo del $\mathrm{LA}^{26}$. Infección cervicovaginal (ICV) con: a) VB o b) cultivo positivo para bacteria patógena primaria o bacteria oportunista en cérvix o vagina (excepto Lactobacillus spp) asociado con incremento de leucocitos polimorfonucleares sobre 10 por campo al examen microscópico directo $(400 x)^{20}$. Se consideró como infección bacteriana ascendente (IBA) la presencia de IMCA por bacterias ascendentes o ICV.

Estudio periodontal. Las mediciones clínicas se hicieron en todos los dientes excepto los terceros molares, por investigadores calibrados. Estas mediciones incluyeron: determinación del nivel de inserción clínica (NIC), profundidad al sondaje periodontal (PS), índice de acumulación de la placa bacteriana supragingival (IP) y determinación del porcentaje de sitios con sangrado al sondaje (SS). Se examinaron 6 sitios por cada diente.

Se consideró como enfermedad periodontal, la periodontitis crónica y la gingivitis. El diagnóstico de periodontitis crónica se hizo ocupando criterios internacionales y usando valores de la profundidad del saco/bolsa periodontal y del NIC. La severidad de la periodontitis se estableció con los valores del NIC y PS en leve, moderada y avanzada o severa (a lo menos cinco o seis dientes con sitios con PS $\geq 5 \mathrm{~mm}, \mathrm{NIC} \geq 3 \mathrm{~mm}$ y extensa pérdida de hueso en la radiografía). Se diagnosticó gingivitis con $\mathrm{SS} \geq 25 \%$ de los sitios, ausencia de saco periodontal y sin pérdida de NIC patológica. La severidad de la gingivitis se midió por el índice gingival (valores numéricos hasta tres): leve $(<1)$, moderado (hasta 2$)$ y severo $(>2)$. Según la extensión la enfermedad periodontal se clasificó en localizada ( $<30 \%$ de sitios comprometidos) o generalizada ( $>30 \%$ de sitios comprometidos $)^{27}$. Las pacientes no recibieron tratamiento periodontal al momento del examen dental.

Se consideró que no hubo periodontitis crónica ni gingivitis en ausencia de sacos periodontales, sin pérdida de NIC y la existencia de un porcentaje de sitios con SS $<25 \%$.

Muestra microbiológica subgingival. Se tomaron dos muestras de la placa bacteriana subgingival de los 4 cuadrantes de la boca, sitios afectados con periodontitis o con gingivitis o sin enfermedad periodontal. Después de aislar con rollos de algodón y de secar con jeringa de aire, se eliminaron los depósitos de tártaro supragingival con curetas tipo Gracey. Luego se colocaron dos conos de papel estéril por $20 \mathrm{~s}$ en cada uno de los sitios seleccionados. Los 8 conos correspondientes a los 4 sitios, se ubicaron en un tubo conteniendo 
el medio de transporte Reduced Transport Fluid

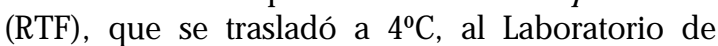
Microbiología, Facultad de Odontología, Universidad de Chile.

Procedimientos microbiológicos periodontales. Las muestras de placa subgingival y de LA, mediante agitación en vortex-mixer fueron dispersadas durante $45 \mathrm{~s}$ para posteriormente hacer diluciones seriadas en RTF. Alícuotas de $100 \mu \mathrm{l}$ de las diluciones apropiadas $\left(10^{-3}, 10^{-4}\right.$, y $\left.10^{-5}\right)$ se sembraron para aislamiento e identificación de microorganismos según metodología descrita por Slots $^{28}$. El diagnóstico diferencial se realizó mediante el empleo de galerías comerciales, (BBL Crystal, Becton-Dickinson). Adicionalmente, para ratificar la presencia de los patógenos periodontales se empleó RPC convencional, con primers hechos en Bios Chile, de acuerdo a técnicas descritas por Ashimoto y cols ${ }^{28}$. Estos estudios se hicieron diferidos. Las muestras se guardaron a $-20^{\circ} \mathrm{C}$.

Para corroborar la identificación, los amplicones se compararon con un banco de datos para la cepa más control positivo y no se secuenciaron.

Se consideró como positivo el cultivo de la placa subgingival cuando el aislamiento bacteriano fue mayor de: Porphyromonas gingivalis $1 \%$, Eikenella corrodens 1\%, Fusobacterium nucleatum 5\%, Prevotella intermedia 2,5\%, Peptostreptococcus micros $5 \%$, Veillonella spp $10 \%$ y Campylobacter rectus $2 \%$.

Manejo de las pacientes. Todas las embarazadas recibieron dos dosis de betametasona $12 \mathrm{mg} \mathrm{IM}$, cada $24 \mathrm{~h}$ y antibióticos: clindamicina $600 \mathrm{mg}$ IV cada 8 h por 48 h y gentamicina $4 \mathrm{mg} / \mathrm{kg} /$ día IV en una dosis por $48 \mathrm{~h}$. Luego se continuó con clindamicina $300 \mathrm{mg}$ oral cada $6 \mathrm{~h}$ por 5 días y gentamicina $4 \mathrm{mg} / \mathrm{kg} /$ día intramuscular en una dosis por 5 días. La utilidad de este esquema está demostrada en publicación anterior de estos autores $^{30}$. Pacientes con cultivo vaginal positivo para Streptococcus agalactiae se trataron con ampicilina o penicilina durante el parto.

Las embarazadas con parto prematuro y MI recibieron fenoterol 0,5 a $3 \mu \mathrm{g} / \mathrm{min}$ IV hasta alcanzar reposo uterino. Se discontinuó con dilatación cervical $>3 \mathrm{cms}$, RPM o infección ovular clínica. Las pacientes que frenaron el parto, se controlaron en el Policlínico del Alto Riesgo materno perinatal hasta las 37 semanas de gestación.

La gestación se interrumpió por cesárea en presencia de: infección ovular clínica o corioamnionitis clínica (definida según los criterios de Gibbs y $\operatorname{cols}^{31}$ ) cultivo de LA positivo para S agalactiae, $\mathrm{N}$ gonorrhoeae, $\mathrm{H}$ influenzae, E coli 0 F nucleatum, sufrimiento fetal, prolapso de cordón y desprendimiento prematuro de placenta.

En las pacientes con RPM y sin IMCA se mantuvo conducta expectante hasta las 35 semanas completas con monitorización electrónica fetal, perfil biofísico, hemograma, proteína C reactiva y cultivos de LA y endocervical semanales. En estos casos, la vía del parto se escogió de acuerdo a las condiciones obstétricas.

Estudio anátomo-patológico placentario. El examen histopatológico fue realizado según técnicas previamente descritas ${ }^{32}$, por un sólo patólogo que estuvo ciego a la información clínica y microbiológica.

Marcadores histológicos de infección. Corioamnionitis: presencia de neutrófilos con o sin necrosis, en membranas fetales o placa subcoriónica. Funisitis: inflamación en el cordón umbilical representada por existencia de neutrófilos en la pared de los vasos con o sin compromiso de la gelatina de Wharton. Se asocia con vasoespasmo y trombosis. La corioamnionitis y funisitis son lesiones agudas y marcadores histológicos de infección ascendente. Vellositis: presencia de neutrófilos en capilares fetales y en el estroma de la vellosidad. Intervellositis, perivellositis: presencia de neutrófilos, linfocitos, macrófagos, ocasionalmente eosinófilos y fibrina en el espacio intervellositario ${ }^{13-16}$. Tanto vellositis como intervellositis y perivellositis pueden ser agudas, pero suelen ser crónicas y aparecer de preferencia en las infecciones transplacentarias.

Expresión de resultados. Se estableció la frecuencia de enfermedad periodontal clínica (periodontitis crónica, gingivitis), la extensión (localizada, generalizada), la severidad (leve, moderada, avanzada o severa); la frecuencia de infección bacteriana ascendente (ICV, IMCA) y la asociación entre enfermedad periodontal e infección bacteriana ascendente.

ARtíCULO DE 
Se describieron las diferentes bacterias aisladas del LA y cervicovaginales, así como los patógenos periodontales aislados del LA y de la placa bacteriana subgingival.

Se estableció la relación entre enfermedad periodontal, IBA y parto prematuro $(<36,6$ semanas) y su expresión en los marcadores histológicos placentarios.

Estadística. Se usaron la prueba de chi cuadrado y la prueba exacta de Fisher en la comparación de proporciones y t de Student o prueba de MannWhitney en la comparación de variables continuas, según correspondiese. Un valor de $\mathrm{p}<0,05$ fue considerado significativo.

\section{Resultados}

Características de la población. En este estudio de cohorte se enrolaron 59 pacientes. Se excluyeron 4 pacientes: dos casos de parto prematuro con MI sin estudio periodontal, un caso con RPM y falla en la obtención de LA y un caso con RPM y malformación fetal.

En la Tabla 1 se describen las características generales de la población.

Enfermedad periodontal. En la Tabla 2 se describe frecuencia, extensión y severidad de la enferme- dad periodontal y su asociación con infección bacteriana ascendente (IBA). La frecuencia de la serie fue $93,2 \%(55 / 59)$.

Microbiología. Se presenta en la Tabla 3. El 27,1\% (16/59) de las pacientes tuvo invasión microbiana cavidad amniótica (IMCA) (9 casos con bacterias periodontales, 5 con bacterias ascendentes y 2 con asociación entre estos dos tipos de bacterias) y 83,1\% (49/59) presentaron infección cervicovaginal (ICV) (incluye 16 casos de IMCA con ICV). La IBA fue $83,1 \%$ [(49/59) incluye 7 casos de IMCA con bacterias ascendentes)]. VB fue 23,7\% (14/59). No hubo IMCA ni ICV en 16,9\% (10/59) de los casos. En 18,6\% (11/59) el cultivo de LA fue positivo para odontopatógenos y en 13,6\% (8/59) para P gingivalis. El $71,2 \%$ de las pacientes $(42 / 59)$ tuvieron cultivo positivo para patógenos periodontales en placa subgingival y 52,5\% (31/59) para P gingivalis. En todos los casos con cultivo positivo en LA para bacterias periodontales, se aisló similar microorganismo en la placa subgingival. En 13 pacientes con enfermedad periodontal clínica, los cultivos en este sitio fueron negativos. En 7 de ellas las muestras se tomaron entre 24 y $72 \mathrm{~h}$ después de iniciado el tratamiento antibiótico.

Parto prematuro. Menor de 37 semanas ocurrió en $64,4 \%$ (38/59). Se asoció significativamente con enfermedad periodontal generalizada $82,1 \%$ (23/

Tabla 1. Características de las pacientes con trabajo de parto prematuro o con rotura prematura de membranas

\begin{tabular}{|lc|}
\hline & $\mathrm{n}=59$ \\
\hline Edad materna (años) & \\
$\quad$ Media (DS) & $26,0(7,69)$ \\
Rango & $16-44$ \\
Paridad & $29(49,2 \%)$ \\
$\quad$ Primípara & $30(50,8 \%)$ \\
Multípara & $30,7(5,4)$ \\
Edad gestacional ingreso (s) & $25,4-34$ \\
Media (DS) & $42(71,2 \%)$ \\
Rango & $17(28,8 \%)$ \\
Casos & \\
Con membranas intactas & \\
\hline
\end{tabular}

$\mathrm{RPM}$, rotura prematura de membranas. 


\section{Tabla 2. Enfermedad periodontal en pacientes con trabajo de parto prematuro} o con rotura prematura de membranas

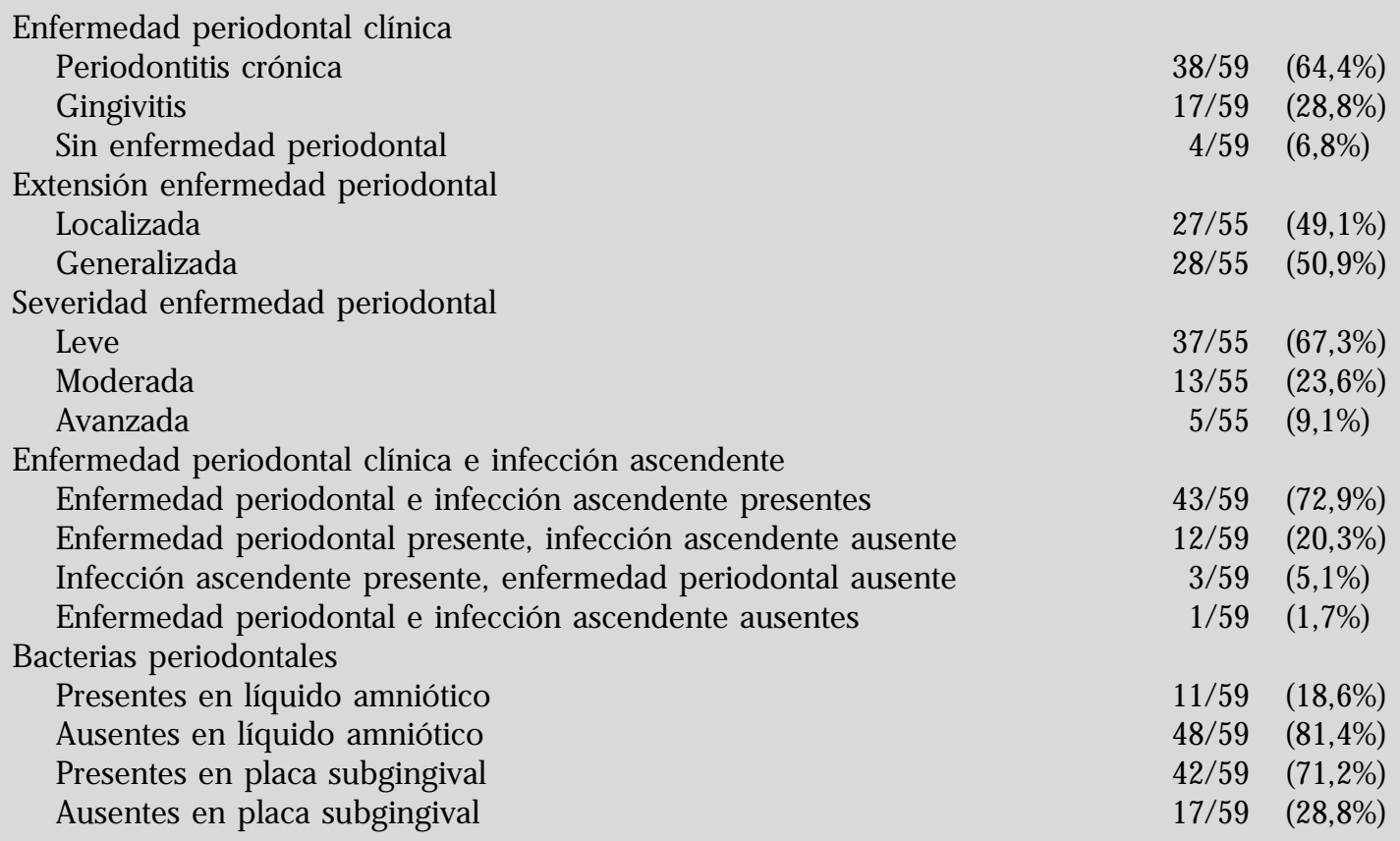

28) vs enfermedad periodontal localizada $48,1 \%$ $(13 / 27)$ y sin enfermedad periodontal $50,0 \%(2 / 4)$ $\mathrm{p}=0,03$. También se presentó significativamente con la asociación IBA-enfermedad periodontal $74,4 \%(32 / 43)$ vs casos sólo con enfermedad periodontal 33,3\% (4/12), casos con infección ascendente solamente $66,6 \%(2 / 3)$ y sin ambas infecciones $p=0,03$. La frecuencia de enfermedad periodontal generalizada y frecuencia de asociación IBA-enfermedad periodontal no fue diferente en los subgrupos con membranas intactas y con RPM. No hubo diferencias en la ocurrencia de parto prematuro en las embarazadas con cultivo positivo y con cultivo negativo para bacterias periodontales aisladas tanto del LA como de la placa subgingival. Tampoco hubo diferencias en prematurez con P gingivalis, la bacteria patógena periodontal más frecuente, cuando se aisló del LA como de la placa subgingival (Tabla 4).

Marcadores histológicos placentarios de infección. Se presentaron sólo en las pacientes con parto prematuro. La $\mathrm{CH}$ se encontró en 57,9\% (22/38), la funisitis en $26,3 \%$ (10/38) y la vellositis, intervellositis, perivellositis en $21,1 \%$ (8/38) de los partos prematuros. La funisitis siempre se encontró asociada con $\mathrm{CH}$. La $\mathrm{CH}$ y funisitis se presentaron en $62,5 \%(20 / 32)$ de los casos con asociación IBAenfermedad periodontal y en las dos pacientes con únicamente infección ascendente. Los cuatro casos con sólo enfermedad periodontal no tuvieron esta patología placentaria (NS). La vellositis e intervellositis aparecieron en $21,9 \%$ (7/32) de las pacientes con asociación y en uno de cuatro casos con únicamente enfermedad periodontal. Los dos casos con infección ascendente no tuvieron vellositis (NS).

Los casos con parto prematuro y enfermedad periodontal generalizada tuvieron una significativa mayor frecuencia de $\mathrm{CH}$ y funisitis $69,6 \%$ (16/ 23) que los casos con enfermedad periodontal localizada $30,8 \%(4 / 13)$ y sin enfermedad periodontal $100 \%(2 / 2) \mathrm{p}=0,04$. En cambio la frecuencia de vellositis, intervellositis, perivellositis en estos grupos no fue significativamente diferente (Tabla 5). 
Tabla 3. M icrobiología de la invasión microbiana de la cavidad amniótica, de la infección cervicovaginal y de la enfermedad periodontal, en pacientes con trabajo de parto prematuro o con rotura prematura de membranas

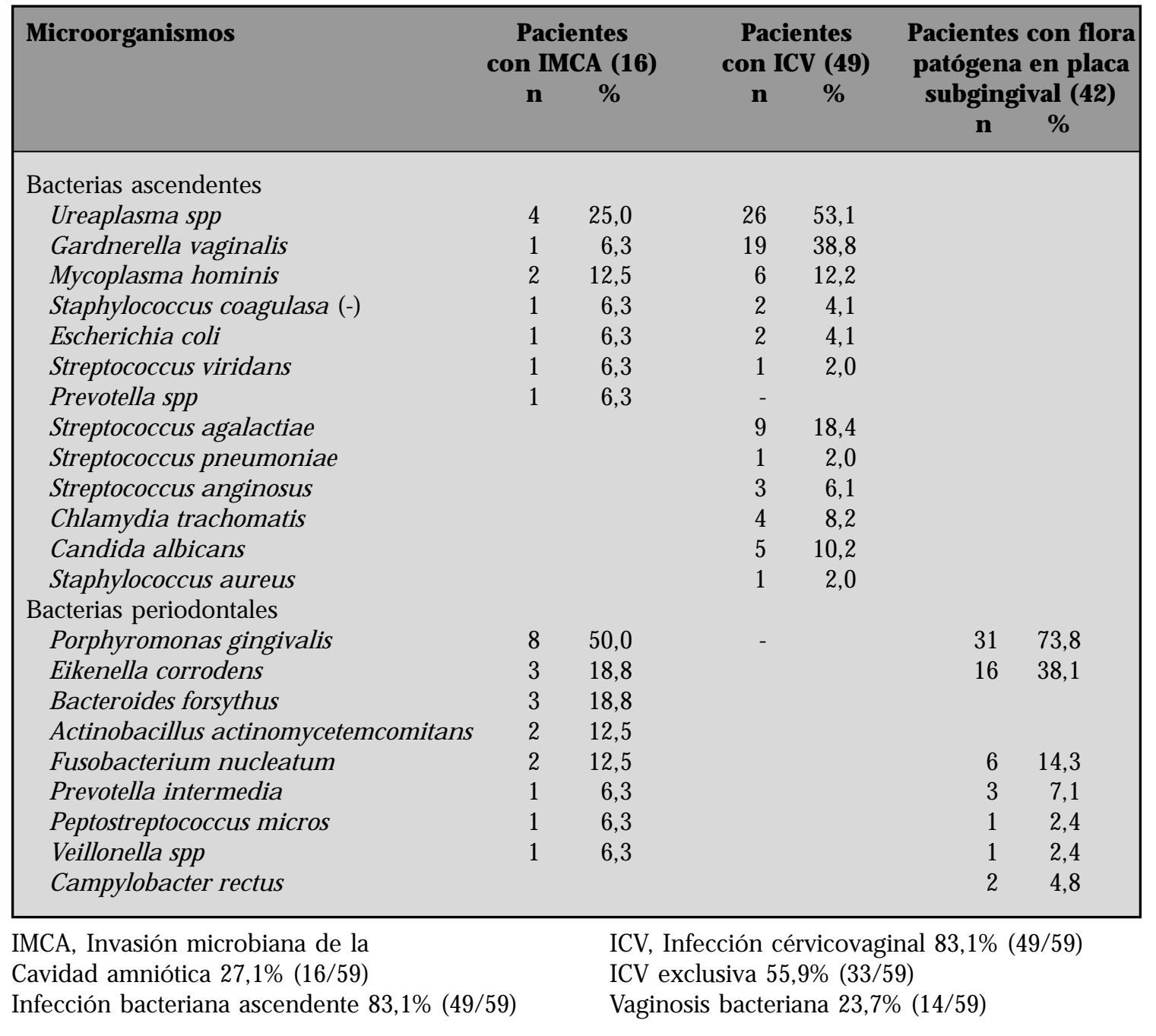

\section{Discusión}

Este estudio sugiere que la enfermedad periodontal generalizada ( $>30 \%$ de sitios comprometidos) y la presencia conjunta de enfermedad periodontal e infección bacteriana ascendente (IMCA-ICV) se asocian con parto prematuro.

Desde 1996 se conoce la relación entre enfermedad periodontal con parto prematuro (MI y RPM) y con niños de bajo peso al nacer, asociación que es independiente de las infecciones genitourinarias ${ }^{12,33}$ y de otros factores de ries- go $^{6,12}$. Desde entonces son variadas las publicaciones que señalan esta relación y veintidós estudios (caso control, cohortes) muestran una positiva asociación ${ }^{34}$.

Este trabajo sugiere que mientras mayor es el compromiso de los sitios con periodontitis, mayor es la asociación con prematurez. Este hallazgo es coincidente con lo publicado en la literatura, que el riesgo de nacimiento prematuro/niños con bajo peso al nacer, es mayor mientras más severa 0 generalizada es la enfermedad periodontal ${ }^{6,9} \mathrm{y}$ que mientras mayor es el progreso de la periodon- 
Tabla 4. Enfermedad periodonal y parto prematuro

\begin{tabular}{|c|c|c|c|c|c|}
\hline $\begin{array}{l}\text { Prematurez y } \\
\text { EP clínica }\end{array}$ & $\begin{array}{l}\text { Periodontitis } \\
\text { crónica } \\
n=38 \\
28(73,7 \%)\end{array}$ & $\begin{array}{l}\text { Gingivitis } \\
n=17 \\
8(47,1 \%)\end{array}$ & $\begin{array}{c}\text { Sin EP } \\
n=4 \\
2(50,0 \%)\end{array}$ & & NS \\
\hline $\begin{array}{l}\text { Prematurez } \\
\text { y extensión EP }\end{array}$ & $\begin{array}{c}\text { EP localizada } \\
n=27 \\
13(48,1 \%)\end{array}$ & $\begin{array}{c}\text { EP generalizada } \\
n=28 \\
23(82,1 \%)\end{array}$ & $\begin{array}{c}\text { Sin EP } \\
n=4 \\
2(50,0 \%)\end{array}$ & & $\begin{aligned} & p \\
= & 0.03\end{aligned}$ \\
\hline $\begin{array}{l}\text { Prematurez } \\
\text { y severidad EP }\end{array}$ & $\begin{array}{c}\text { EP leve } \\
n=37 \\
23(62,2 \%)\end{array}$ & $\begin{array}{c}\text { EP moderada } \\
n=13 \\
9(69,2 \%)\end{array}$ & $\begin{array}{c}\text { EP avanzada } \\
n=5 \\
4(80,0 \%)\end{array}$ & $\begin{array}{c}\text { Sin EP } \\
n=4 \\
2(50,0 \%)\end{array}$ & $\begin{array}{l}\mathrm{p} \\
\mathrm{NS}\end{array}$ \\
\hline $\begin{array}{l}\text { Prematurez } \\
\text { asociación EP } \\
\text { e infección } \\
\text { ascendente }\end{array}$ & $\begin{array}{c}\text { EP e infección } \\
\text { ascendente } \\
\text { presentes } \\
\text { n }=43 \\
32(74,4 \%)\end{array}$ & $\begin{array}{l}\text { EP presente, } \\
\text { infección } \\
\text { ascendente } \\
\text { ausente } \\
n=12 \\
4(33,3 \%)\end{array}$ & $\begin{array}{l}\text { EP ausente, } \\
\text { infección } \\
\text { ascendente } \\
\text { presente } \\
n=3 \\
2(66,6 \%)\end{array}$ & $\begin{array}{c}\text { EP e infección } \\
\text { ascendente } \\
\text { ausentes } \\
\text { n =1 } \\
0\end{array}$ & $=0,03$ \\
\hline $\begin{array}{l}\text { Prematurez } \\
\text { y bacterias } \\
\text { periodontales }\end{array}$ & $\begin{array}{c}\text { Bacterias } \\
\text { periodontales } \\
\text { presentes en LA } \\
n=11 \\
7(63,6 \%)\end{array}$ & $\begin{array}{c}\text { Bacterias } \\
\text { periodontales } \\
\text { ausentes en LA } \\
\mathrm{n}=48 \\
31(64,6 \%)\end{array}$ & $\begin{array}{c}\text { Bacterias } \\
\text { periodontales } \\
\text { presentes en PSG } \\
n=42 \\
27(64,3 \%)\end{array}$ & $\begin{array}{c}\text { Bacterias } \\
\text { periodontales } \\
\text { ausentes en PSG } \\
\mathrm{n}=17 \\
11(64,7 \%)\end{array}$ & NS \\
\hline
\end{tabular}

EP: Enfermedad periodontal, LA: Líquido amniótico, PSG: Placa subgingival

Enfermedad periodontal generalizada MI: 52,4\% (11/21) RPM 70,6\% (12/17) NS

Enfermedad periodontal asociación con infección ascendente M: 76,2\%\% (16/21) RPM 94,1\% (16/17) NS

\section{Tabla 5. Relación entre extensión de la enfermedad periodontal y la histopatología placentaria en pacientes con parto prematuro}

\begin{tabular}{|lcccc|}
\hline $\begin{array}{l}\text { Histopatología } \\
\text { placentaria }\end{array}$ & $\begin{array}{c}\text { Enfermedad } \\
\text { periodontal } \\
\text { localizada } \\
\mathbf{n = 1 3}\end{array}$ & $\begin{array}{c}\text { Enfermedad } \\
\text { periodontal } \\
\text { generalizada } \\
\mathbf{n = 2 3}\end{array}$ & $\begin{array}{c}\text { Sin enfermedad } \\
\text { periodontal }\end{array}$ & $\mathbf{P}$ \\
\hline $\begin{array}{l}\text { Corioamnionitis y funisitis } \\
\text { Vellositis e intervellositis }\end{array}$ & $\begin{array}{r}4(30,8 \%) \\
\mathbf{n}(23,1 \%)\end{array}$ & $\begin{array}{r}16(69,6 \%) \\
5(21,7 \%)\end{array}$ & $\begin{array}{c}2(100 \%) \\
0\end{array}$ & $\begin{array}{c}\text { NS } \\
\text { NS }\end{array}$ \\
\hline
\end{tabular}

titis durante la gestación los resultados adversos del embarazo son más serios y la prematurez es más precoz ${ }^{11}$.

Otro resultado importante de este estudio y no descrito en la literatura es que la presencia conjunta de enfermedad periodontal e infección bacteriana ascendente se asocia con nacimiento prematuro. Esto significa que simultáneamente la microbiota patógena periodontal y la respuesta inflamatoria diseminada por vía hematógena 
transplacentaria, junto con la microbiota patógena ascendente del tracto genital inferior, infectan e inflaman la cavidad amniótica, probablemente se potencian y producen el nacimiento prematuro. Es necesario diseñar trabajos que intenten explicar esta asociación y el momento en que aparecen estas infecciones en el parto prematuro.

La ruta que sigue la enfermedad periodontal para infectar la cavidad uterina es hematógena transplacentaria de acuendo a las evidencias existentes: a) detección aumentada de inmunoglobulinas (IgM) en sangre fetal para $F$ nucleatum y $\mathrm{P}$ gingivalis en niños prematuros ${ }^{35}$; b) detección de $\mathrm{P}$ gingivalis, uno de los odontopatógenos de mayor virulencia, en hígado materno, placenta e hígado fetal luego de implantes subcutáneos en conejas preñadas $^{36}$; c) incremento de la respuesta inflamatoria fetal (IL-1, IL-6, FNT, PE2) y de IgM específicas contra patógenos periodontales y aumento de nacimientos prematuros ${ }^{37} \mathrm{y}$ d) hallazgo de similitud de cepas de $\mathrm{F}$ nucleatum aisladas a nivel subgingival y en LA en embarazadas con parto prematuro ${ }^{38}$.

Entonces, la patología placentaria a encontrar en los partos prematuros asociados con enfermedad periodontal debiera ser vellositis, intervellositis y perivellositis, marcadores histológicos que aparecen de preferencia en las infecciones transplacentarias ${ }^{13-16}$. Sin embargo, en este estudio la frecuencia de $\mathrm{CH}$ y funisitis (marcadores histológicos de infección ascendente) fue significativamente mayor en el grupo con mayor riesgo de parto prematuro (periodontitis generalizada) que en los de menor riesgo (periodontitis localizada), en cambio la frecuencia de vellositis, intervellositis y perivellositis fue similar en estos grupos.

Estos resultados podrían tener las siguientes explicaciones que necesariamente deben ser demostradas por trabajos futuros: 1) el tratamiento antibiótico sistémico (clindamicina-gentamicina) es eficiente en erradicar o disminuir el patógeno periodontal, la respuesta inflamatoria asociada y el daño placentario; 2) el tratamiento antibiótico usado no es tan eficiente en erradicar o disminuir la microbiota de la infección bacteriana ascendente y la patología placentaria producida.

1. Tratamiento antibiótico sistémico y enfermedad periodontal. Se sabe actualmente que la remoción de la placa subgingival por medios mecánicos, ultrasonido u otros es segura y mejora la enfermedad periodontal ${ }^{39}$, pero no modifica significativamente las tasas de nacimientos prematuros o peso pequeño al nacer $^{35,40}$. La Academia Americana de Periodontología establece que los antibióticos sistémicos $^{41}$ (la clindamicina es uno de los recomendados), son eficientes para el tratamiento de la periodontitis severa ${ }^{42}$. En este estudio, la probable acción eficaz de la asociación antibiótica clindamicina-gentamicina en erradicar o disminuir patógenos periodontales y respuesta inflamatoria asociada, podría explicar la baja frecuencia de vellositis, intervellositis y perivellositis encontrada en el grupo con mayor parto prematuro (periodontitis generalizada). Si se comprueba que existe este beneficio, la detección precoz de la periodontitis severa o extensa, conjuntamente con el tratamiento antibiótico sistémico y odontológico de esta enfermedad, podrían disminuir el parto prematuro.

2. Tratamiento antibiótico sistémico y parto prematuro. El tratamiento antibiótico en la RPM de pretérmino retrasa el parto, reduce la morbilidad neonatal ${ }^{43}$ y la incidencia de corioamnionitis histológica ${ }^{32}$; en tanto que en el parto prematuro con MI sin causa clínica evidente, prolonga la gestación, reduce la morbilidad materna, pero no disminuye el parto prematuro ${ }^{44}$. En este trabajo la mayor frecuencia encontrada de corioamnionitis y funisitis histológica en los grupos con mayor incidencia de prematurez, es probable que se deba a que los antimicrobianos usados fueron menos eficientes en disminuir estas patologías placentarias.

Una limitación de este trabajo es el gran número de pacientes no enroladas, por la imposibilidad de hacer estudio microbiológico a todos los casos elegidos y que pudo ser una forma de error de selección de pacientes. Sin embargo, no hay razones para pensar que las embarazadas con trabajo de parto prematuro y membranas intactas o con rotura prematura de membranas se comporten de una manera diferente según el momento en que acuden al hospital (mañanas versus tardes o noches). Más importante aún, este sesgo puede intervenir sobre la prevalencia de ciertos fenómenos, pero no sobre la clara asociación entre la 
presencia de enfermedad periodontal-infección bacteriana ascendente y los resultados del embarazo. Esta situación de embarazadas no enroladas determinó la segunda limitación, impidió tener una casuística más numerosa que habría dado mayor solidez a los resultados. Sin embargo para el resultado principal, la enfermedad periodontal generalizada se asocia con parto prematuro, el poder de la muestra de $68 \%$, es suficiente para sostener esta relación.

\section{REFERENCIAS}

1. Goldenberg RL, Culhane JF, IAms JD, Romero $R$ Epidemiology and causes of preterm birth. Lancet 2008; 371: 75-84.

2. IAms JD, Romero R, Culhane JF, Goldenberg RL Primary, secondary, and tertiary interventions to reduce the morbidity and mortality of preterm birth. Lancet 2008; 371: 164-75.

3. Page RC, Kornman KS. The pathogenesis of human periodontitis: an introduction. Periodontol 2000 1997; 14: 9-11.

4. Van Winkelhoff AJ, Loos BG, Van Der Reijden WA, VAn DeR Velden U. Porphyromonas gingivalis, Bacteroides forsythus and other putative periodontal pathogens in subjects with and without periodontal destruction. J Clin Periodontol 2002; 29: 1023-8.

5. Offenbacher S, Beck JD, LiefF S, Slade G. Role of periodontitis in systemic health: spontaneous preterm birth. J Dent Educ 1998; 62: 852-8.

6. JefFcoat M, Geurs N, Reddy M, Cliver S, Goldenberg R, HaUth J. Periodontal infection and preterm birth: Results of a prospective study. J Am Dent Assoc 2001; 132: 875-80.

7. O fFenbacher S, Jared HL, O'Reiluy PG, Wells SR, Salvi GE, Lawrence HP ET aL. Potential pathogenic mechanisms of periodontitis associated pregnancy complications. Ann Periodontol 1998; 3: 233-50.

8. Moss M, Beck J, Genco R, Salvi G, Offenbacher S. Progressing periodontitis is associated with increased Serum Tumor Necrosis Factor Alpha (TNF alpha). J Dent Res 1995; 74: 158-63.

9. Goepfert AR, JefFcoat MK, Andrews WW, Faye-PeterSen O, Cliver SP, Goldenkerg RL et al. Periodontal disease and upper genital tract inflammation in early spontaneous preterm birth. Obstet Gynecol 2004; 104: 777-83.

10. Offenbacher S, LiefF S, Boggess KA, Murtha AP, Madianos PN, Champagne CM ET AL. Maternal periodontitis and prematurity. Part I: Obstetric outcome of prematurity and growth restriction. Ann Periodontol 2001; 6: 164-74.
En conclusión, la enfermedad periodontal generalizada y la presencia conjunta de enfermedad periodontal e infección bacteriana ascendente se asocian con parto prematuro. En estos casos, son frecuentes los marcadores histológicos placentarios de infección ascendente. Futuros estudios se requieren para determinar si el uso de antibióticos sistémicos en la embarazada con enfermedad periodontal disminuye el nacimiento prematuro.

11. Offenbacher S, Boggess KA, Murtha AP, Jared HL, LeFF S, McKaIG RG, et aL. Progressive periodontal disease and risk of very preterm delivery. Obstet Gynecol 2006; 107: 29-36.

12. Offenbacher S, Katz V, Fertik G, Cowins J, Boyd D, Maynor G ET AL. Periodontol 1996; Periodontal infection as a possible risk factor for preterm low birth weight. J Periodontol 1996; 67: 1103-13.

13. Bernischke K, Kaufman P. Pathology of the human placenta. Chapter: Histopathological Approach to Villous Alteration, 419-34. Chapter: Infectious diseases, 591-659. Nework NY: Springer Verlag NY, Inc; 2001.

14. PERRIN VDK. Pathology of the Placenta. Chapter: Placenta as a Reflection of maternal disease, 57-70. New York, Churchill Livingstone Inc; 1984.35. Fox H. Pathology of the Placenta. Chapter: Histological Abnormalities of the Placenta, 149-97. Philadelfia, Pa: WB Saunders Co; 1978.

15. KeeLung JW. Fetal and Neonatal Pathology. Chapter: Placenta and Umbilical Cord p 45-67 SpringerVerlag. Berlin Heidelberg 1987.

16. Van Hoeven KH, Anyaegbunam A, Hochster H, Whitty JE, Distant J, CRawford C ET aL Clinical significance of increasing histologic severity of acute inflammation in the fetal membranes and umbilical cord. Pediatric Pathology 1996; 16: 731-44.

17. Juez G, Lucero E, Ventura-Junca P, Tapia JL, Winter A. Crecimiento intrauterino en recién nacidos chilenos de clase media. Rev Chil Pediatr 1989; 60: 198-202.

18. Taylor-Robinson D, FurR PM. Recovery and identification of genital tract Mycoplasmas. Israel J Med Sci 1981; 17: 648-53.

19. Claas HC, Melchers WJ, De Bruju IH, De Graaf M, VAN DiJK WC, Lindeman J ET AL. Detection of Chlamydia trachomatis in clinical specimens by the polymerase chain reaction. Eur J Clin Microbiol \& Infect Dis 1990; 9: 864-8.

20. Ovalue A, Martínez MA, Giglo MS, Poblete P, Fuentes A, Viliablanca E. Microbiología aislada en la rotura prematura de membranas de pretérmino. Relación con morbilidad infecciosa materna neonatal e inter-

ARtíCULO DE 
valo rotura de membranas-parto. Rev Chil Obstet Ginecol 1995; 60: 252-62.

21. Wang E, Richardson H. A rapid method for detection of group B streptococcal colonization: testing at the bedside. Obstet Gynecol 1990; 76: 882-5.

22. Viliagra E, Martínez MA, Ovaue A. Flora microbiana genital en una población de alto riesgo obstétrico. Rev Chil Obstet Ginecol 1994; 59: 32-8.

23. Murray PR, Baron EJ, Jorgensen JH, Pfaler MA, YolKen RH (Eds). Manual of Clinical Microbiology. $8^{\text {th }}$ Ed, 2003 American Society for Microbiology, Washington DC

24. Ovalue A, Martínez MA, Gómez R, Sáez J, Menares I, AspiLaga $\mathrm{C}$ ET al. Parto prematuro con membranas intactas. Microbiología del líquido amniótico y tracto genital inferior y su relación con los resultados maternoneonatales. Rev Méd Chile 2000; 128: 985-95.

25. Nugent R, KRohn M, Huшer S. Reliability of diagnosing bacterial vaginosis is improved by standardized method of Gram stain interpretation. J Clin Microbiol 1991; 29: 297-301.

26. Romero R, Mazor M, Morrotti R, Avila C, Oyarzún E, INSUNZA A ET AL. Microbial invasion of the amniotic cavity in spontaneous rupture of membranes at term. Am J Obstet Gynecol 1992; 166: 129-33.

27. Armitage GC. Development of a classification system for periodontal diseases and conditions. Annals of Periodontology 1999; 4: 1-6.

28. Sцотs J. Rapid identification of important periodontal microorganims by cultivation. Oral Microbiol Immunol 1986; 1: 48-57.

29. Аshimoto A, Chen C, Bakker I, SLots J. Polymerase chain reaction detection of 8 putative periodontal pathogens in subgingival plaque of gingivitis and advanced periodontitis lesions. Oral Microbiol Immunol 1996; 11: 266-73.

30. Ovalue A, Martínez MA, Gómez R, Rubio R, Fuentes A, VALDERRAMA O. Antibiotic therapy in patients with preterm premature of membranes: a prospective, randomized, placebo controlled study with microbiologic assessment of the amniotic cavity and lowergenital tract. Prenat Neonat Med 1997; 2: 21322.

31. Gibbs RS, CastiLo MS, Rogers PJ. Management of acute chorioamnionitis. Am J Obstet Gynecol 1980; 136: 109-13.

32. Ovalue A, Martínez Ma, Kakarieka E, Gómez R, Rubio $\mathrm{R}$, VALDERRAMA $\mathrm{O}$ ET AL. Antibiotic administration in patients with preterm premature rupture of membranes reduces the rate of histological chorioamnionitis: a prospective, randomized, controlled study. Journ Mat Fet Neonat Med 2002; 12: 35-41.

33. Vergnes JN, Sixou M. Preterm low birth weight and maternal periodontal status: a meta-analysis. Am J Obstet Gynecol 2007; 196: 135. e1-7.

34. Clothier B, Stringer M, JefFcoat MK. Periodontal disease and pregnancy outcomes: exposure, risk and intervention. Best Practice \& Research Clinical Obstetrics and Gynaecology 2007; 21: 451-66.

35. Madianos PN, LiefF S, Murtha AP, Boggess KA, Auten RL JR, BECK JD ET AL. Maternal periodontitis and prematurity. Part II: Maternal infection and fetal exposure. Ann Periodontol 2001; 6: 175-82.

36. Boggess KA, Madianos PN, Preisser JS, Moise KJ JR, OfFENBACHer S. Chronic maternal and fetal Porphyromonas gingivalis exposure during pregnancy in rabbits. Am J Obstet Gynecol 2005; 192: 554-7.

37. Boggess KA, Moss K, Madianos P, Murtha AP, Beck J, OFFENBACHER S. Fetal immune response to oral pathogens and risk of preterm birth. Am J Obstet Gynecol 2005; 193: 1121-6.

38. HIL GB. Preterm birth: associations with genital and possibly oral microflora. Ann Periodontol 1998; 3: 222-32.

39. Forgie A, Beirne P, Worthington HV, Ciarkson Je. Routine scale and polish for periodontal health in adults (Protocol for a Cochrane Review). The Cochrane Library 2004.

40. Michalowicz BS, Hodges JS, Diangelis AJ, Lupo VR, Novak MJ, Ferguson JE ET AL, FOR THE OPT Study. Treatment of Periodontal Disease and the Risk of Preterm Brith. N Engl J Med 2006; 355: 1885-94.

41. Sьots J. Research, Science and Therapy Committee. Systemic antibiotics in periodontics. J Periodontol 2004; 75: 1553-65.

42. Gordon J, Walker C, Hovliaras C, Socransky S. Efficacy of clindamycin hydrochloride in refractory periodontitis: 24-month results. J Periodontol 1990; 61: 686-91.

43. Kenyon S, Boulvain M, Neilson J. Antibiotics for preterm rupture of membranes (Cochrane Review). In: The Cochrane Library, Issue 4, 2007. Oxford: Update Software.

44. KING J, FlenAdy V. Prophylactic antibiotics for inhibiting preterm labour with intact membranes. Cochrane Database Syst Rev. 2002; (4): CD000246. 